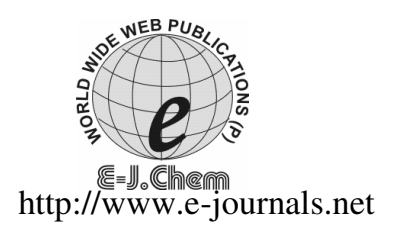

ISSN: 0973-4945; CODEN ECJHAO

E-Journal of Chemistry

2011, 8(3), 1298-1308

\title{
Thermodynamic and Calorimetric Study of Acetylsalicylic Acid (Aspirin) and Ibuprofen
}

\author{
JUAN CARLOS MORENO-PIRAJÁN*, VANESSA SILENIA GARCÍA-CUELLO \\ and LILIANA GIRALDO-GUTIERRÉZ ${ }^{\S}$
}

\author{
*Grupo de Investigación en Sólidos Porosos y Calorimetría \\ Departamento de Química, Universidad de los Andes \\ Carrera 1 No 18 A 10, Bogotá/Colombia \\ ${ }^{\S}$ Facultad de Ciencias, Departamento de Química \\ Universidad Nacional de Colombia \\ Carrera 30 No 45-03, Bogotá/Colombia \\ jumoreno@uniandes.edu.co
}

Received 16 June 2010; Revised 23 November 2010; Accepted 15 December 2010

\begin{abstract}
Enthalpies of solution and dilution of aqueous solutions of sodium acetylsalicylic acid salt and ibuprofen salt were measured with an isoperibolic calorimeter at $293.15 \mathrm{~K}, 298.15 \mathrm{~K}, 303.15 \mathrm{~K}, 308.15 \mathrm{~K}$ and $318.15 \mathrm{~K}$. The concentration of the electrolyte was restricted to the solubility of the salt at various temperatures and did not exceed $0.035-0.057 \mathrm{~mol} \mathrm{~kg}^{-1}$, depending on the temperature studied. The Virial coefficients were derived from Pitzer's model and the excess thermodynamic functions of both the solution and the components of the solution were calculated. An analysis of the thermodynamic characteristics of the solution in terms of concentration and temperature interval was carried out and discussed. Additionally, an analysis was performed by differential scanning calorimetry (DSC).
\end{abstract}

Keywords: Acetylsalicylic acid, Ibuprofen, Differential scanning calorimetry, Solution calorimetry, Descomposition, Thermogravimetry

\section{Introduction}

Several investigations have been carried out on the application of thermal analysis for investigating aspirin and ibuprofen in the presence of tablet excipients. The identification of non-prescription internal analgesics has been investigated by means of thermogravimetry and differential thermal analysis (TG and DTA) ${ }^{1}$. Studies of acetylsalicylic acid ${ }^{2}$, mixtures of acetylsalicylic acid and $p$-chloroaniline ${ }^{3}$, aspirin ${ }^{4,5}$ and ibuprofen have also been investigated. In the present work, aspirin was studied using TG-DTA, DSC and solution calorimetry to elucidate its thermal decomposition mechanism. 
Solution (dilution) enthalpy is an important thermodynamic function and may be used effectively for the characterization of different properties of drug molecules ${ }^{4-6}$. For example, solution enthalpies have been used to describe the crystallinity of amorphous materials in $\operatorname{drugs}^{7}$ and to analyse various energetic drug-solvent interaction terms ${ }^{8,9}$. There are some studies devoted to studying the heat effects of the nucleation process in order to obtain information about the stability of pharmaceutical solutions ${ }^{10-12}$. Moreover, in the literature, a semi-empirical approach proposed by Pitzer $^{13,14}$ and applied by other authors such as Manin's et al. ${ }^{15,16}$ describes long and short distance interionic interactions of salts in solutions. This approach provides an opportunity to consider the thermochemical data within the framework of the model for both concentration and temperature intervals and to calculate whole sets of excess solution thermodynamic functions based only on thermochemical experiments. In the present study, the sodium salts of acetylsalicylic acid and ibuprofen were chosen as the subjects of investigation (Figure 1) because this compound is a widely-used, non-steroidal anti-inflammatory drug and several of its properties have been discussed in the literature ${ }^{8,13,14,16}$.
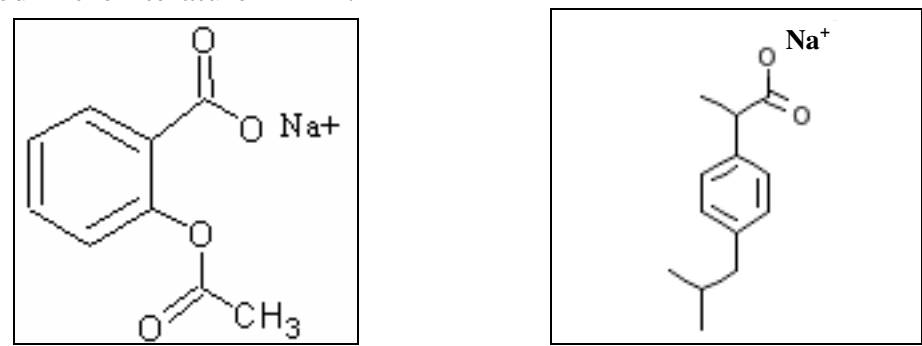

Figure 1. Structural formulae of the sodium salts of acetylsalicylic acid and ibuprofen

However, the solvation characteristics of this substance have not yet been studied and this fact is a barrier between analysing the outlined properties on the one side and pharmacodynamic and pharmacokinetic data on the other. This work presents the results of a thermodynamic study on the sodium salts of acetylsalicylic acid and ibuprofen in aqueous solutions under wide concentration and temperature intervals (important for pharmaceutical and medical reasons) and a thermodynamic characterization of this system using Pitzer's approach ${ }^{13-15}$ with the procedure presented by Manin et al. ${ }^{16}$ for comparison with our results.

\section{Experimental}

Acetylsalicylic acid (aspirin) was obtained from Bayer Germany) and the ibuprofen sodium salt (Na-IB) was a pure-grade product (Sigma-Aldrich, US) and used without further purification. The DSC curves were obtained using a Netzsch $409 \mathrm{C}$ thermoanalyzer system with an air drying flux of $110 \mathrm{~mL} \mathrm{~min}^{-1}$, a heating rate of $10 \mathrm{~K} \mathrm{~min}^{-1}$ and with the sample weighing about $0.10 \mathrm{mg}$. An aluminium crucible with a perforated cover was used for the DSC.

\section{Isoperibol calorimeter and materials}

Reagent grade hydrochloric acid (Merck) and the water used in the dissolution measurements were produced through Milli-Q filtration. This system was used to prepare a stock solution of $0.10047 \mathrm{M} \mathrm{HCl}$. This solution was standardized with tris(hydroxymethyl) aminomethane (THAM) (Merck, Analytic Reagent, 99.999\%) and then checked against standard sodium hydroxide solutions; $0.2000 \mathrm{~g}$ in each case and a $\mathrm{pH}$ meter was used to detect the end points. To compare the results of this work, the samples were prepared as described 
in the literature, especially taking into account the suggestions of the standards committee of the U.S. Calorimetry conference ${ }^{17,18}$ to determine the solution heat of reaction of THAM(s) with $\mathrm{HCl}$ using specific experimental conditions, including:

- The reaction temperature should be maintained at $298.15 \mathrm{~K}$.

- The concentration of THAM should be $5 \mathrm{~g} \mathrm{~L}^{-1}$.

- The concentration of the $\mathrm{HCl}$ solution should be 0.100 mole $\mathrm{L}^{-1}$.

- The THAM sample should be stored with no further crushing or grinding in an atmosphere of 50\% relative humidity, weighed in air and sealed in a glass ampoule with atmospheric pressure.

- A blank determination should be made.

These conditions were followed in this study. The values for the heat of the reaction of THAM(s) with $0.100 \mathrm{M} \mathrm{HCl}$ were measured at $298.15 \mathrm{~K}$. The THAM used to measure the heat reaction was prepared following the previous procedure ${ }^{18}$; a thin layer of this sample was spread across a watch glass and maintained at $298.15 \mathrm{~K}$ in a desiccator over a saturated magnesium nitrate solution which provided an atmosphere of about $50 \%$ relative humidity.

\section{Operational mode of the isoperibol calorimeter}

The THAM samples (approximately $0.1387 \mathrm{~g}$ ) were weighed in air and sealed with a small membrane of paraffin at atmospheric pressure and coupled to the dual-pneumatic valves system (for the dosing of liquids or solids) of the high-precision calorimeter used in this work. For comparison, some ampoules were carefully sealed in the same shape to simulate the opening of the valve and the system of mixture. Each sample was mixed in a solution of $50 \mathrm{~mL} \mathrm{HCl}$, leaving a final solution concentration of $5 \mathrm{~g} \mathrm{~L}^{-1}$ of THAM and the mixture was computer controlled from outside the developed system.

\section{Solution calorimetry}

\section{Device design and operation}

The instrument used in this research was a single isothermal micro-calorimeter that uses a specially constructed thermistor system which produces a voltage proportional to the heat flow between the dissolution cell and the surroundings ${ }^{18}$. The calorimeter is laid out in a water bath, where the temperature is maintained at $\pm 6 \times 10^{-4} \mathrm{~K}$. The water bath, temperature controller and all of the peripheral units were built in our laboratory and can be seen in Figure 2.

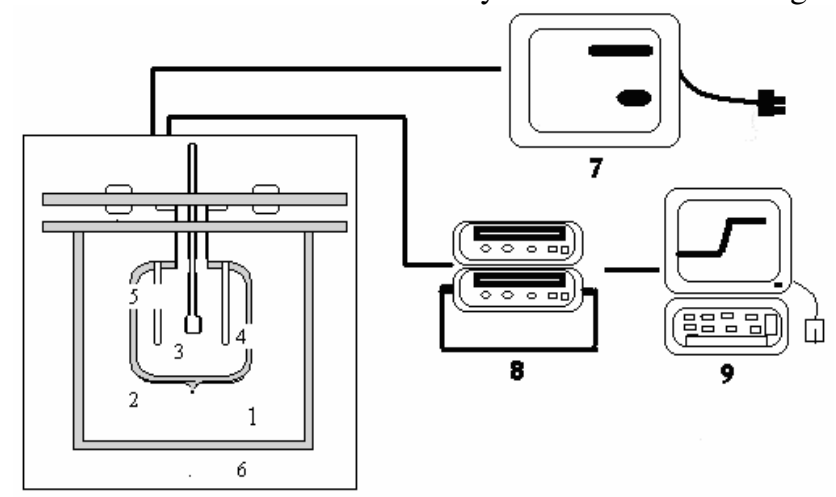

Figure 2. Dissolution isoperibol microcalorimeter general schematic diagram, (1 Internal jacket, 2 Dewar vessel, 3 Glass ampoule, 4 Thermistors, 5 Calibration resistance, 6 External jacket, 7 Breaking control, 8 Multimeters, 9 Interphase capture data) 
The cell consists of a Dewar-type bottle glass (1) that has been vacuum-sealed $\left(10^{-8} \mathrm{mBar}\right)$ and whose volume is $50 \mathrm{~mL}$. The cell possesses a flange containing a channel fitting for an $o$-ring, thus ensuring a perfect hermetic seal with the top lid that contains the elements which communicate with the peripheries. A coating was specially designed for this machine that allows the reflux of water at $298.15 \mathrm{~K}$. The coating was manufactured using carbon fibre (2), which not only provides insulation but converts the calorimeter into a robust machine. This unit is fitted with a glass lid that is also covered with carbon fibre, affixed by a set of precision screws (3).

This equipment has platinum resistance to convey $30 \Omega$ electric calibrations (4) and the temperature sensor system is based on the thermistor system described below (5). The mixture system consists of two parts: a pneumatic valve, externally controlled by a computer, with a solution kept at a temperature of $\pm 0.0001 \mathrm{~K}$ and which allows one of the mixture solvents in question to be injected and the mixing system, which is a counter-clockwise blade made of stainless steel (6). The electronic system encompasses hardware features such as the data acquisition card based on a microcontroller connected to a PC via RS232 through a serial port $^{18}$. The digitalization of the thermometric signal is performed as described in the ${ }^{18}$, while leaving room to allow a basic system to perform virtual treatments, which is new for this type of experiment. The system could be programmed by the user in order to capture the signals and to apply certain mathematical equations to the signals obtain the required information.

\section{Calculation procedure}

All calculations were carried out on the basis of experimental data from the solution enthalpies of sodium acetylsalicylic acid salt and ibuprofen salt labelled, Na-AA and Na-IB, respectively, in water $\left(\Delta_{\text {sol }} \mathrm{H}_{\mathrm{m}}\right)$ and the molar dilution enthalpy $\Delta_{\text {dil }} \mathrm{H}^{\mathrm{mi}-\mathrm{mf}}$ of the concentrated solution from the initial $\left(\mathrm{m}_{\mathrm{i}}\right)$ to final $\left(\mathrm{m}_{\mathrm{f}}\right)$ concentrations:

$$
\Delta_{\text {sol }} H^{m_{i}}=\Delta_{\text {sol }} H^{m_{f}}-\Delta_{\text {dil }} H^{m_{i} \rightarrow m_{f}}=\Delta_{\text {sol }} H^{m_{f}}-L_{\Phi}
$$

Where L $\phi$ is a relative partial molar enthalpy of the solution electrolytes (Na-AA and $\mathrm{Na}-\mathrm{IB}$ ) at molality $\mathrm{m}$. On the other hand, the L $\phi$ value can be described from Pitzer's model $^{13,14}$ and Manin's model ${ }^{16}$ as:

$$
L_{\Phi}=\left|z_{M} z_{X}\right|\left(2 A_{H} / 3 b\right) \ln \left(1+b I^{1 / 2}\right)-2 v_{M} v_{X} R T^{2}\left(m B_{M X}^{\prime}+m^{2} C_{M X}^{\prime}\right)
$$

where:

$$
\begin{gathered}
B_{M X}^{\prime}=\left(\partial \beta_{M X}^{(0)} / \partial T\right)_{I, P}+\left(2 / \alpha^{2} I\right)\left(\partial \beta_{M X}^{(1)} / \partial T\right)_{I, P}\left[1-\left(1+\alpha I^{1 / 2}\right) \exp \left(-\alpha I^{1 / 2}\right)\right] \\
C_{M X}^{\prime}=0.5\left(v_{M} v_{X}\right)^{1 / 2}\left(\partial C_{M X}^{\Phi} / \partial T\right)_{I, P}
\end{gathered}
$$

Where $\mathrm{I}$ is the ionic strength, $\mathrm{zM}$ and $\mathrm{zX}$ are the charges of the cations and anions, respectively, $\mathrm{M}$ and $\mathrm{X}$ are the number of cations and anions in the electrolyte molecules (Na_AA and Na-IB), respectively and $\mathrm{A}_{\mathrm{H}}$ is the theoretical slope of enthalpy (limit of the Debye-Hückel slope) and is borrowed from Manin's model ${ }^{16}$. The first term of Eq. (2) corresponds to long distance electrostatic interactions. The second term corresponds to short-distance interactions and includes the various Virial coefficients: $\beta^{0}{ }_{M X}, \beta^{1}{ }_{M X}$ and $C^{\phi}{ }_{M X}$. The b and $\alpha$-values were chosen as 1.2 and $2.0 \mathrm{~kg}^{1 / 2} \mathrm{~mol}^{1 / 2}$, respectively, as recommended in the literature method ${ }^{10,19}$. The temperature dependencies of the Virial coefficients were interpolated by the equations presented in the next section (Tables 3 and 4) using the model described in $^{19}$. The equations used ${ }^{19-21}$ to describe the thermodynamic functions of the excess solution were: 


$$
\begin{aligned}
H^{E} & \equiv L_{\Phi}=-\Delta_{d i l} H^{m \rightarrow 0} \\
\overline{L_{1}} & =-0.5 m^{3 / 2} M_{1}\left(\partial L_{\Phi} / \partial m^{1 / 2}\right) \\
\overline{L_{2}} & =L_{\Phi}+0.5 m^{1 / 2}\left(\partial L_{\Phi} / \partial m^{1 / 2}\right) \\
\Phi\left(T_{2}\right)-\Phi\left(T_{1}\right) & \left.=\left(m^{1 / 2} / 2 v R\right) \int_{T_{1}}^{T_{2}}\left[\partial L_{\Phi} / \partial m^{1 / 2}\right) d(1 / T)\right] \\
\ln \left[\gamma\left(T_{2}\right) / \gamma\left(T_{1}\right)\right] & =(1 / v R) \int_{T_{1}}^{T_{2}}\left[L_{\Phi}+\left(m^{1 / 2} / 2\right)\left(\partial L_{\Phi} / \partial m^{1 / 2}\right) d(1 / T)\right] \\
C_{P}^{E} & =\overline{C_{P}}-C_{P}^{0}=\left(\partial L_{\Phi} / \partial T\right)_{P}
\end{aligned}
$$

Where $\phi$ and $\gamma$ are osmotic and activity coefficients, respectively; $\bar{L}_{1}$ and $\bar{L}_{2}$ are the partial molar enthalpies of the solvent and solute of the studied solutions; $v$ is the number of ions of Na-AA (=2) and Na-IB (=2); M 1 is the molar mass of water; $C_{P}^{E}$ is the excess heat capacity of the solution under constant pressure.

\section{Results and Discussion}

The value determined in this study for the heat of the reaction at $25^{\circ} \mathrm{C}$ with THAM(s) in $\mathrm{HCl}$ solution concurs with previously reported values at this temperature and under similar experimental conditions within a range of $\pm 0.01 \%$. The value obtained in this study for the heat of reaction at $298.15 \mathrm{~K}$ with THAM(s) in an $\mathrm{HCl}$ solution, together with other reported values including those at the same values reported for other published studies $^{18,19}$, was $-17.29 \mathrm{~kJ} \mathrm{~mol}^{-1}$. This showed the proper functioning ${ }^{18}$ of the equipment built and that it could be used for the thermodynamic studies proposed in this research.

The DSC curves, Figures $3 a$ and $3 b$, were endothermic, as both salts can be seen. The first endothermic peak of aspirin at $453.15 \mathrm{~K}$ followed by the second endothermic peak at $553.15 \mathrm{~K}$ were due to fusion, followed by the elimination of acetic acid and accompanied by the evaporation of acetylsalicylic and salicyclic acids. Ibuprofen showed the first peak at $368.15 \mathrm{~K}$ and the second one at 573.15 , both endothermic.

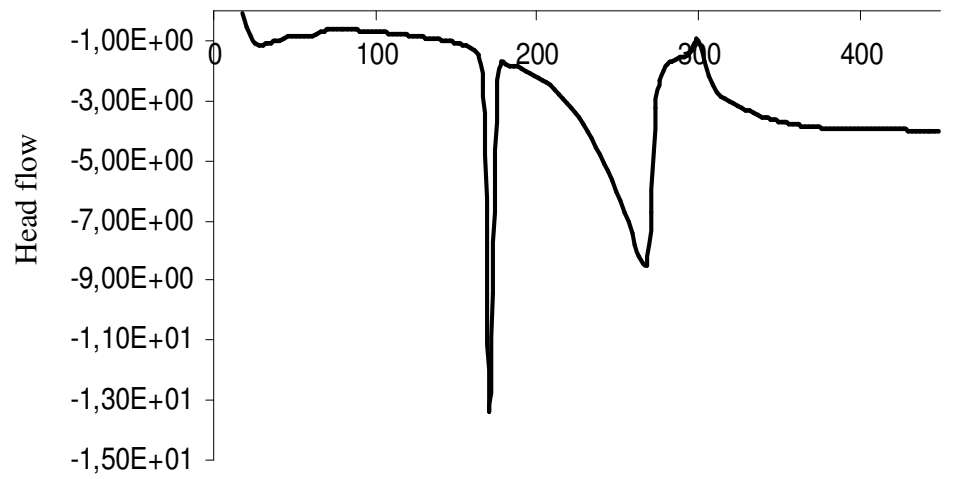

Temperature, $\mathrm{C}$

Figure 3a. DSC of aspirin 


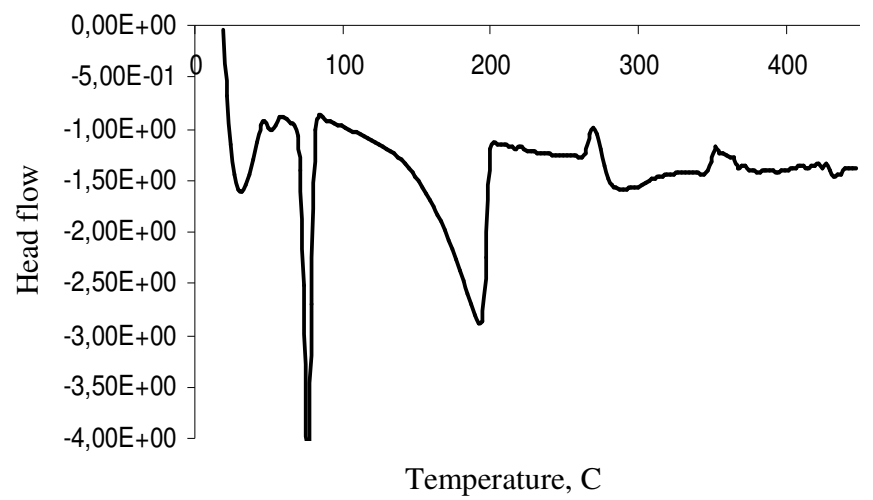

Figure 3b. DSC of Ibuprofen

The enthalpy for ibuprofen was $98.84 \mathrm{~J} \mathrm{~g}^{-1}$, which agrees with the results of similar studies in the literature ${ }^{18-22}$. The enthalpy of aspirin was $134.42 \mathrm{~J} \mathrm{~g}^{-1}$. These results show purity of the drugs. The experimental data of the solution and dilution enthalpies of Na-AA and Na-IB in water at various concentrations and temperatures are presented in Table 1 and Figure 4.

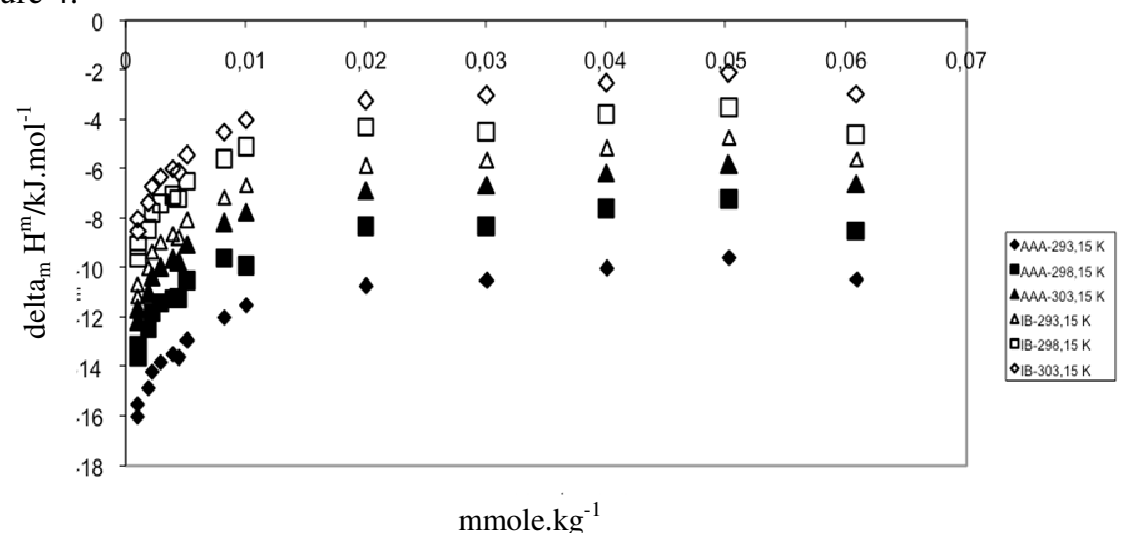

Figure 4. Concentrations dependencies of $\mathrm{Na}-\mathrm{AA}$ and $\mathrm{Na}-\mathrm{IB}$ solution enthalpies in water at different temperatures

As can be seen from Figure 4, the endothermic effect increased with increasing concentrations of both salts up to $0.02 \mathrm{~mol} \mathrm{~kg}^{-1}$ and with decreasing temperature. It should be mentioned that the function of the heat effects from the concentration is practically independent of concentrations higher than $0.03 \mathrm{~mol} \mathrm{~kg}^{-1}$. It is interesting to note that the $\mathrm{Na}-\mathrm{AA}$ and Na-IB solution enthalpies decreased with increasing temperature. It can be assumed that the solvation process of the studied ionic mixture was determined by a cavernous mechanism. As the temperature increased, the primary water structure was destroyed and became a more closely packed structure: as a consequence, an endothermic cost of creating an appropriate cavity volume in the solvent for the solute molecules arose. This same behaviour and interpretation for the salt of aspirin was reported by other authors $^{14,16}$. In this work, however, the endothermic effect was more marked than in other studies, which we attributed to the concentrations used in this work and the sensitivity of the calorimeter, which was higher here. 
Table 1. Dilution enthalpies, $-\Delta_{\mathrm{dil}} \mathrm{H}^{\mathrm{mi} \rightarrow \mathrm{mf}}, \mathrm{kJ}^{-1} \mathrm{~mol}^{-1}$, of Na-AA and Na-IB in water at various temperatures

\begin{tabular}{|c|c|c|c|c|c|}
\hline \multicolumn{2}{|c|}{$\mathrm{m}_{\mathrm{i}} / \mathrm{mol} \mathrm{kg}^{-1}$} & \multicolumn{2}{|c|}{$\mathrm{m}_{\mathrm{f}} / \mathrm{mol} \mathrm{kg}^{-1}$} & \multicolumn{2}{|c|}{$-\Delta_{\mathrm{dil}} \mathrm{H}^{\mathrm{mi} \rightarrow \mathrm{mf}}$} \\
\hline $\mathrm{Na}-\mathrm{AA}$ & Na-IB & $\mathrm{Na}-\mathrm{AA}$ & $\mathrm{Na}-\mathrm{IB}$ & Na-AA & $\mathrm{Na}-\mathrm{IB}$ \\
\hline \multicolumn{6}{|c|}{$\mathrm{T}=293.15 \mathrm{~K}$} \\
\hline 0.01556 & 0.02347 & 0.00056 & 0.00087 & 3.056 & 3.176 \\
\hline 0.03154 & 0.03576 & 0.00324 & 0.00387 & 3.234 & 3.567 \\
\hline 0.03188 & 0.03599 & 0.00109 & 0.00178 & 3.818 & 4.038 \\
\hline 0.03216 & 0.03878 & 0.00245 & 0.00289 & 4.165 & 4.498 \\
\hline 0.03577 & 0.04012 & 0.00098 & 0.00112 & 4.309 & 4.989 \\
\hline 0.03605 & 0.04123 & 0.00100 & 0.01347 & 4.967 & 5.187 \\
\hline \multicolumn{6}{|c|}{$\mathrm{T}=298.15 \mathrm{~K}$} \\
\hline 0.01468 & 0.01675 & 0.00087 & 0.00088 & 2.879 & 3.345 \\
\hline \multicolumn{6}{|c|}{$\mathrm{T}=303.15 \mathrm{~K}$} \\
\hline 0.03212 & 0.03443 & 0.00099 & 0.00102 & 2.187 & 2.345 \\
\hline 0.03687 & 0.03984 & 0.00067 & 0.00089 & 2.987 & 2.891 \\
\hline 0.03656 & 0.03945 & 0.00054 & 0.00067 & 2.387 & 2.798 \\
\hline 0.03654 & 0.03912 & 0.00189 & 0.00045 & 2.190 & 2.345 \\
\hline 0.04306 & 0.04567 & 0.00198 & 0.00078 & 2.089 & 2.237 \\
\hline 0.04309 & 0.04587 & 0.0201 & 0.00107 & 1.998 & 2.098 \\
\hline 0.04308 & 0.04567 & 0.0209 & 0.00145 & 2.34 & 2.657 \\
\hline \multicolumn{6}{|c|}{$\mathrm{T}=310.15 \mathrm{~K}$} \\
\hline 0.02789 & 0.03125 & 0.00087 & 0.00096 & 2.781 & 2.981 \\
\hline 0.02788 & 0.03134 & 0.00064 & 0.00134 & 2.244 & 2.172 \\
\hline 0.04678 & 0.04865 & 0.00152 & 0.00176 & 2.198 & 2.187 \\
\hline 0.04699 & 0.04789 & 0.00187 & 0.00243 & 2.182 & 2.374 \\
\hline
\end{tabular}

In order to estimate the excess thermodynamic functions, the temperature independent $\mathrm{q}_{i}$ parameters were calculated. The results are summarized in Tables 2 and 3. Dependencies of the relative characteristics of the activity and osmotic coefficients on concentration are presented in Figure 5. It should be noted that the outlined functions were not linear. Moreover, the function $\phi_{\mathrm{T}}-\phi_{293.15}=\mathrm{f}(\mathrm{m})$ had an inversion point at $0.029 \mathrm{~m}$.

Table 2. Temperature independent parameters, $q_{i}$, for calculating the $L_{\phi}$ values of $\mathrm{Na}-\mathrm{AA}$ and Na-IB

$$
\begin{aligned}
& \begin{array}{ccc}
\hline \text { Parameters }^{\mathrm{a}} & \mathrm{Na}-\mathrm{AA} & \mathrm{Na}-\mathrm{IB} \\
\hline \mathrm{q} 2 & (2.78 \pm 0.46) .10^{6} & (3.35 \pm 0.56) .10^{6}
\end{array} \\
& \text { q3 } \quad(2.34 \pm 0.19) \cdot 10^{4} \quad(3.04 \pm 0.34) \cdot 10^{4} \\
& \text { q4 } \quad-36.9 \pm 3.8 \quad-56.6 \pm 7.9 \\
& \text { q9 } 13.8 \pm 3.7 \\
& \text { q10 (23.8 } \pm 1.6) \cdot 10^{-3} \quad\left(34.6 \pm 2.7=10^{-3}\right. \\
& { }^{a} \beta_{M X}^{(0)}=q_{1} \frac{\mathrm{s}\left(\mathrm{L}_{\phi}\right)}{+q_{2}\left[\left(T_{R}-T\right) / T T_{R}\right]+q_{3} \ln \left(T / T_{R}\right)+q_{4}\left(T-T_{R}\right)+q_{5}}\left(T^{2}-T_{R}^{2}\right) \text {; } \\
& \beta_{M X}^{(1)}=q_{6}+q_{9}\left(T-T_{R}\right)+q_{10}\left(T^{2}-T_{R}^{2}\right) \text {; } \\
& C_{M X}^{\Phi}=q_{11}+q_{12}\left[\left(T_{R}-T\right) / T T_{R}\right]+q_{13} \ln \left(T / T_{R}\right)+q_{14}\left(T-T_{R}\right) \\
& \text { where } T_{R}=298 \mathrm{~K}
\end{aligned}
$$


Table 3. The $\left(\partial \beta_{M X}^{(0)} / \partial T\right)_{I, P}^{a}$ and $\left(\partial \beta_{M X}^{(1)} / \partial T\right)_{I, P}^{b}$ values for calculating the $\mathrm{L}_{\phi}$ values of

$\mathrm{Na}-\mathrm{AA}$ and $\mathrm{Na}-\mathrm{IB}$ in water at various temperatures ${ }^{\mathrm{c}}$

\begin{tabular}{|c|c|c|c|c|c|c|}
\hline $\mathrm{T}, \mathrm{K}$ & $\partial \beta_{M X}^{(0)} / \partial T$ & 1 & $\left(\partial \beta_{M X}^{(1)} / \partial T\right.$ & / & \multicolumn{2}{|c|}{$\mathrm{s}\left(\mathrm{L}_{\phi}\right) \mathrm{J} \mathrm{mol}^{-1}$} \\
\hline 293.15 & $0.756 \pm 0.115$ & $0.865 \pm 0.124$ & $1.115 \pm 0.024$ & $1.453 \pm 0.0034$ & 546 & 660 \\
\hline 303.15 & $0.673 \pm 0.124$ & $0.798 \pm 0.134$ & $1.005 \pm 0.032$ & $1.254 \pm 0.0045$ & 509 & 590 \\
\hline 310.15 & $0.604 \pm 0.109$ & $0.702 \pm 0.145$ & $0.967 \pm 0.034$ & $1.113 \pm 0.023$ & 498 & 560 \\
\hline 315.15 & $0.587 \pm 0.110$ & $0.678 \pm 0.156$ & $0.876 \pm 0.0045$ & $1.054 \pm 0.0036$ & 468 & 516 \\
\hline 320.15 & $0.509 \pm 0.112$ & $0.587 \pm 0.167$ & $0.743 \pm 0.0013$ & $0.987 \pm 0.014$ & 440 & 478 \\
\hline
\end{tabular}

${ }^{a}\left(\partial \beta_{M X}^{(0)} / \partial T\right)_{I, P}=-q_{2} / T^{2}+q_{3} / T+q_{4}+2 q_{5} T ;$

${ }^{b}\left(\partial \beta_{M X}^{(1)} / \partial T\right)_{I, P}=q_{9}+2 q_{10} T$

${ }^{c}\left(\partial C_{M X}^{\Phi} / \partial T\right)_{I, P}=-q_{12} / T^{2}+q_{13} / T+q_{14}=0$ within the studied temperature interval

The minimal value of the activity coefficient corresponded to $0.028 \mathrm{~m}$, whereas for the osmotic coefficient it corresponded to $0.019 \mathrm{~m}$. Dependencies of the excess solution heat capacities $\left(C_{P}^{E}\right)$ were evaluated at various concentrations, but the graphs are not shown here.

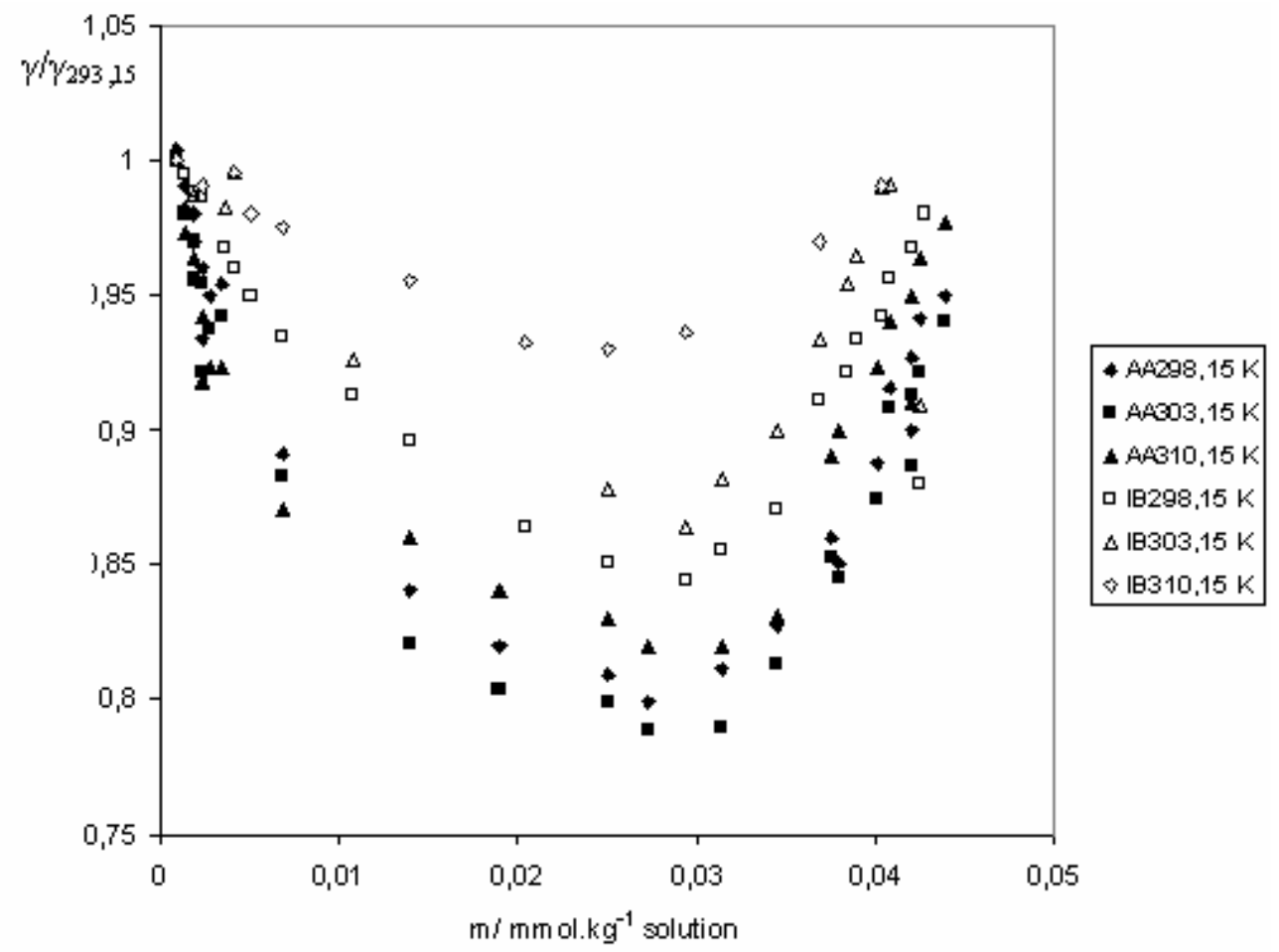




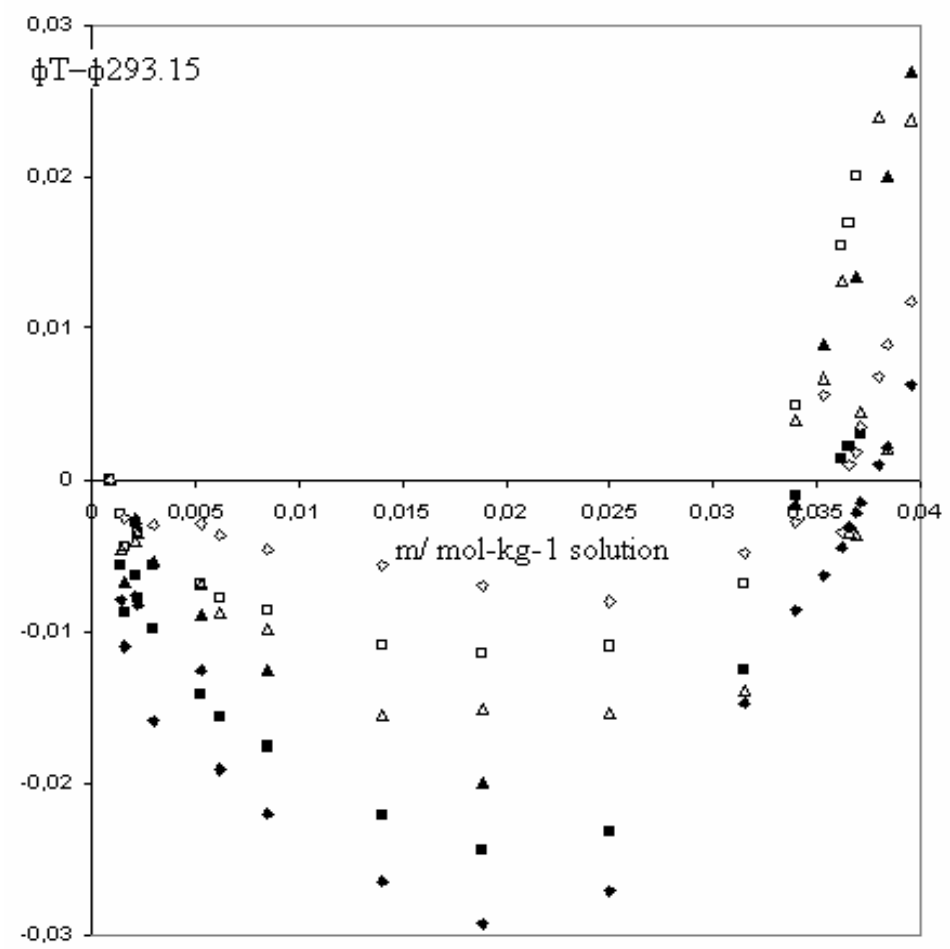

Figure 5. Dependences of a $\gamma / \gamma_{293.15 \mathrm{~K}}$ and $\phi_{\mathrm{T}}-\phi_{293.15} v \boldsymbol{s}$. Na-AA and Na-IB concentrations at various temperatures

These results showed a linear trend between temperature and inversion intervals of around $305.15-310.15 \mathrm{~K}$. This regularity testifies that the temperature factor has an essential influence on the structure of the studied solution. Moreover, as the concentration of the solution increases, the $C_{P}^{E}$ function becomes more sensitive to temperature variations. This result was modified with respect to temperature and was associated with the interaction between the respective salts of the medicines used in this research project and this behaviour agrees with previous reports in the literature.

In order to analyse the behaviour of the solution in detail, the relative partial molar enthalpies for the water $\left(\bar{L}_{1}\right)$ and both Na-AA and Na-IB $\left(\bar{L}_{2}\right.$, respectively) were considered $^{16}$. Let us first consider the thermodynamic properties of the water molecules in solution. The results of the calculations of the $\overline{L_{1}}=\mathrm{f}(\mathrm{m})$ function at the various temperatures are shown in Figure 6. It should be mentioned that at low Na-AA and Na-IB concentrations, the structure of the water molecules in solution was essentially rebuilt: the $\overline{L_{1}}$ values changed from -2000 to $-5000 \mathrm{~J} \mathrm{~mol}^{-1}$. The $\overline{L_{1}}=\mathrm{f}(\mathrm{m})$ function was minimal at concentrations around $0.035-0.048 \mathrm{~mol} \mathrm{~kg}^{-1}$ (depending on T). Minimum functioning was found in the region of the lower Na-AA and Na-IB concentrations, at 293.15-303.15 K, whereas at 310.15 K the minimum shifted to the interval with higher Na-AA and Na-IB concentrations. At $\mathrm{m}<0.035$ mol kg${ }^{-1}$, there was an increase in the interactions between Na-AA and Na-IB molecules 
with the water molecules in the solution (structuring effect): negative $\bar{L}_{1}$ values (Figure 4). An increase in both salt concentrations led to a decrease in the interactions between the solvent molecules in the solution (disordering effect): positive $\bar{L}_{1}$ values (Figure 4). This behaviour is identical to that reported by other authors such as Manin et al ${ }^{16}$.

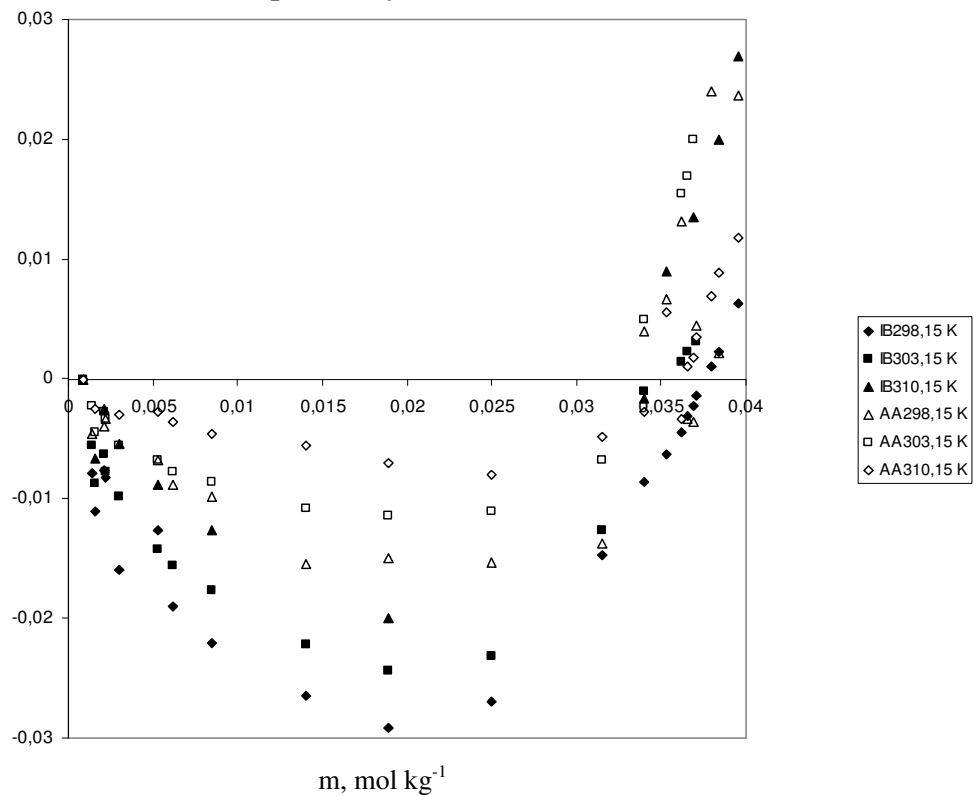

Figure 6. Dependences of $\overline{L_{1}}$ vs. Na-AA and Na-IB concentrations at various temperatures

For the next step, let us analyse the behaviour of the solute molecules in solution. The $\bar{L}_{2}=\mathrm{f}(\mathrm{T})$ and $\bar{L}_{2}=\mathrm{f}(\mathrm{m})$ dependencies were mirror images of $\overline{L_{1}}$, therefore the Figures are not presented here. The maximum $\bar{L}_{2}=\mathrm{f}(\mathrm{m})$ function corresponded to a concentration interval of $0.035-0.048 \mathrm{~mol} \mathrm{~kg}^{-1}$. The region with $\mathrm{m}<0.035 \mathrm{~mol} \mathrm{~kg}^{-1}$ describes the weak hydration of the two salts with water, whereas at $\mathrm{m}>0.035 \mathrm{~mol} \mathrm{~kg}^{-1}$ hydration increased, probably due to both the destruction of the primary water structure and the association between solute molecular processes.

The temperature coefficients $\frac{\overline{\partial L_{1}}}{\partial T}$ and $\frac{\partial L_{2}}{\partial T}$ (Figure 4) had different signs: at $\mathrm{T}<303.15: \mathrm{K}$ $\overline{\frac{\partial L_{1}}{\partial T}}>0$ and $\overline{\frac{\partial L_{2}}{\partial T}}<0$ and at $\mathrm{T}>308.15 \mathrm{~K}: \frac{\overline{\partial L_{1}} \mathrm{~T}}{\partial T}<0$ and $\frac{\partial \overline{L_{2}}}{\partial T}>0$. In other words at $\mathrm{T}<303.15 \mathrm{~K}$ $\overline{C_{P 1}}>\overline{C_{P 1}^{0}}$ and $\overline{C_{P 2}}<\overline{C_{P 2}^{0}}$ (structuring effect of the water molecules in the solution), whereas at $\mathrm{T}>308.15 \mathrm{~K} \overline{C_{P 1}}>\overline{C_{P 1}^{0}}$ and $\overline{C_{P 2}}<\overline{C_{P 2}^{0}}$ (disordering effect of water in the solution for the salts of both drugs). It should be noted, that at the extreme point $\left(\overline{C_{P 1}} \approx \overline{C_{P 1}^{0}}\right)$ the partial molar heat capacity of water molecules in the solvent was equal to the pure water heat capacity and that $\overline{C_{P 2}} \approx \overline{C_{P 2}^{0}}$ corresponded to equal values of the salts partial molar heat capacities in solution and at infinite dilution of drugs. 


\section{Conclusion}

In view of the results of the DSC, it can be seen that it is a desirable tool for the chemical analysis of drugs, as well as for examining the purity of drugs. The complete set of thermodynamic characteristics of Na-AA and Na-IB aqueous solutions were investigated over the whole concentration and physiologically important temperature intervals. The particular concentration of the studied solution where the osmotic coefficients were not dependent on temperature (inversion point) was established. The three concentration intervals of the solution under investigation where the hydration characteristics of the salts of the drugs investigated essentially differed from each other were defined.

\section{Acknowledgment}

The authors would like to thank the Departments of Chemistry at the Universidad Nacional de Colombia and the Universidad de Los Andes (Colombia) and the Master Agreement established between these two institutions. Special thanks go to the Fondo Especial de Investigaciones de la Facultad de Ciencias de la Universidad de Los Andes (Colombia) for its financing.

\section{References}

1. Acquistucci R, Bucci R, Magrì A.D and Magrì A.L, Thermochim Acta, 1991, 188, 51-60.

2. Magrì A L, Balestrieri F, Magrì A D and Marini D, Talanta, 1995, 42, 1719-1723.

3. Balestrieri F, Magrì A D, Magrì A L, Marini D and Sacchini A, Thermochim Acta, 1996, 285, 337-345.

4. Acquistucci R, Bucci R, Magrì A D, Magrì A L and Fresenius J, Anal Chem., 1997, 357, 97-100.

5. Magrì A D, Magrì A, Balestrieri F, Sacchini A, Marini D and Fresenius J, Anal Chem., 1997, 357, 985-988.

6. Bucci R, Magrì A D, Magrì A L and Fresenius J, Anal Chem., 1998, 362(7-8), 557-582.

7. $\quad$ Craig D Q M and Newton J M, Int J Pharm., 1991, 74(1-2), 43-48.

8. Grant D J W, York P, Int J Pharm., 1986, 28, 103-112.

9. Gao D and Rytting, J.H, Int J Pharm., 1997, 151, 183-192.

10. Fini A, Fazio G and Feroci G, Int J Pharm., 1995, 126, 95.

11. Lloyd G R, Craig D Q M and Smith A, Eur J Pharm Biopharm., 1999, 48, 59-65.

12. Pudipeddi M, Sokoloski T D, Duddu S P and Carstensen J T, J Pharm Sci., 1995, 84(10), 1236-1239.

13. Pitzer K S, J Chem Phys., 1973, 77, 268-276.

14. Pitzer K S, Thermodynamic Modeling for Geology: Minerals, Fluids and Alloys, 1992, 110.

15. Chadha R, Kashid N and Jain D V S, J Pharm Biomed Anal., 2003, 30(5), 1515-1522.

16. Manin N G, Fini A, Manin A N and Perlovich G L, J Therm Anal Cal., 2007, 90, 147-152.

17. Wadso I., Thermochim Acta, 1995, 267, 45-49.

18. Moreno-Piraján J C and Giraldo L, J Therm Anal Cal., 2009, 96, 853-857.

19. Silvester L F and Pitzer K S, J Phys Chem., 1977, 81, 1822-1831.

20. Busey R H, Holmes H F and Mesmer R E, J Chem Thermodyn., 1984, 16, 343-372.

21. Mayrath J E and Wood R H, J Chem Thermodyn., 1983, 15, 625-632.

22. Bucci R, Magri A.D and Magri A.L, J Therm Anal Cal., 2000, 61, 369-376. 


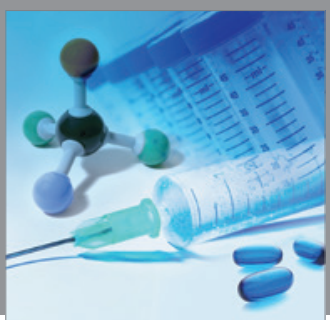

International Journal of

Medicinal Chemistry

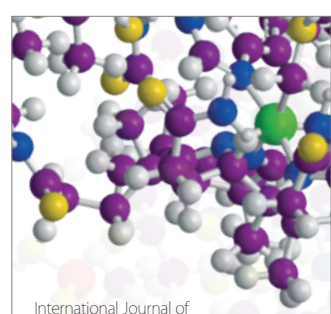

Carbohydrate Chemistry

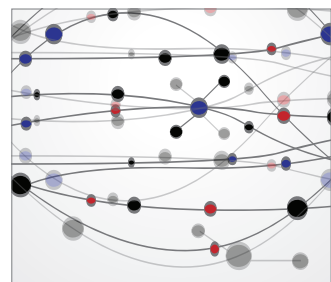

The Scientific World Journal
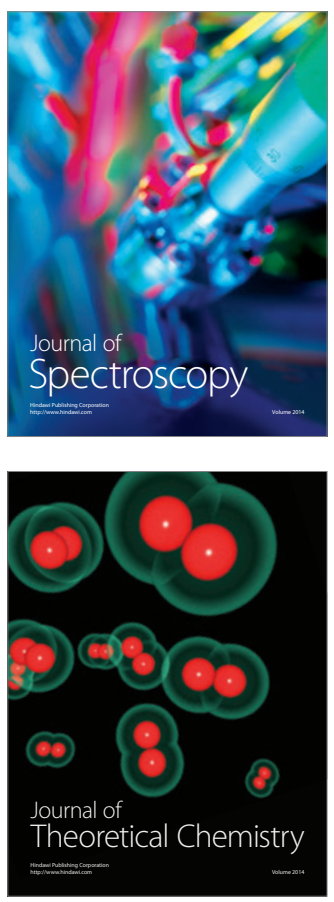
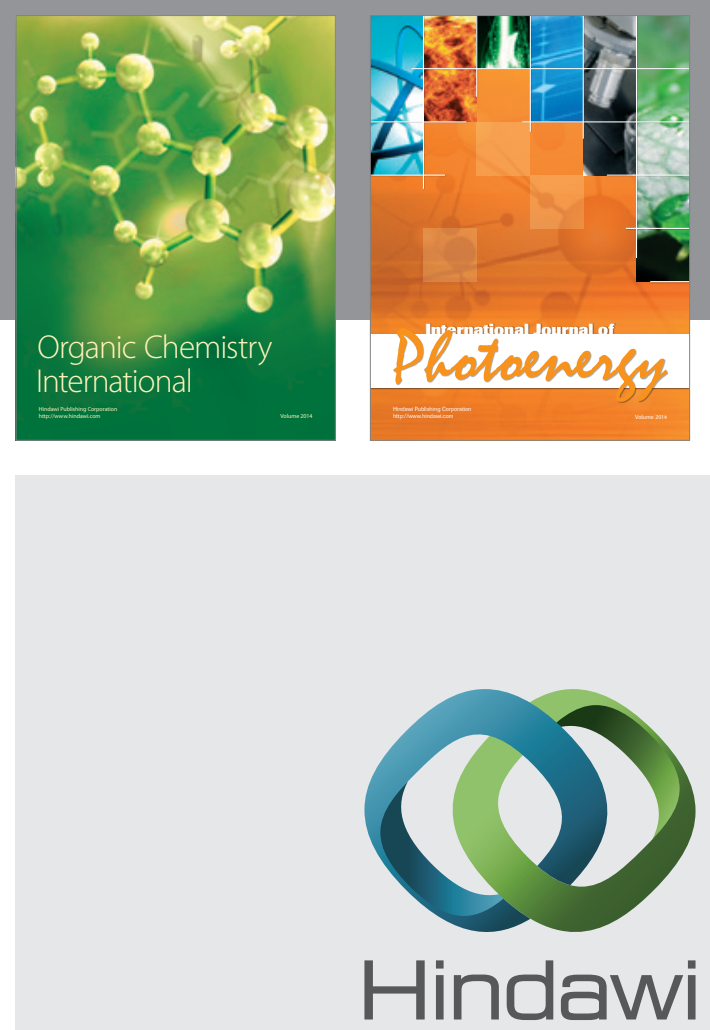

Submit your manuscripts at

http://www.hindawi.com
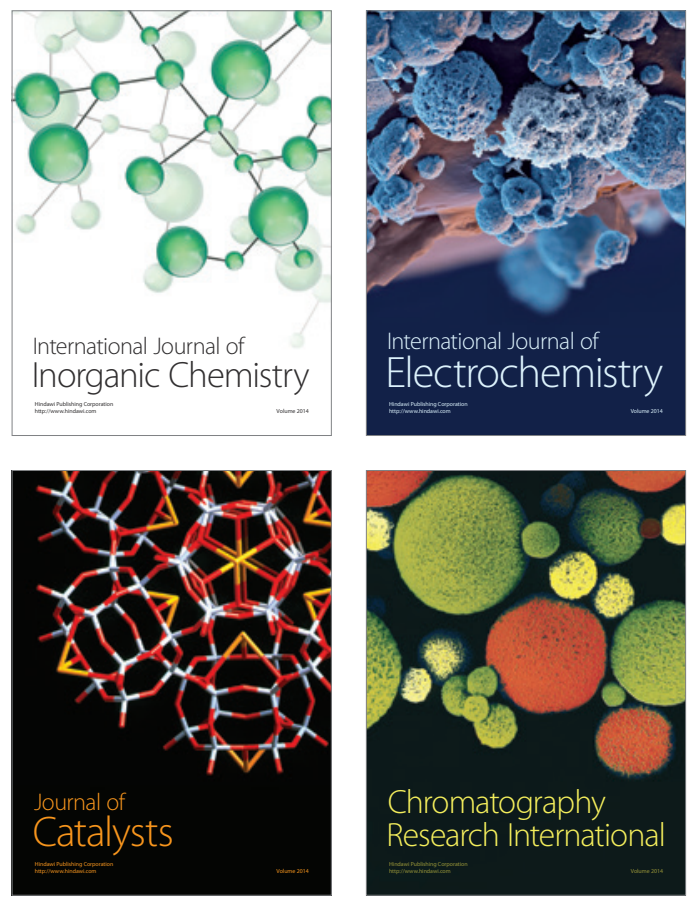
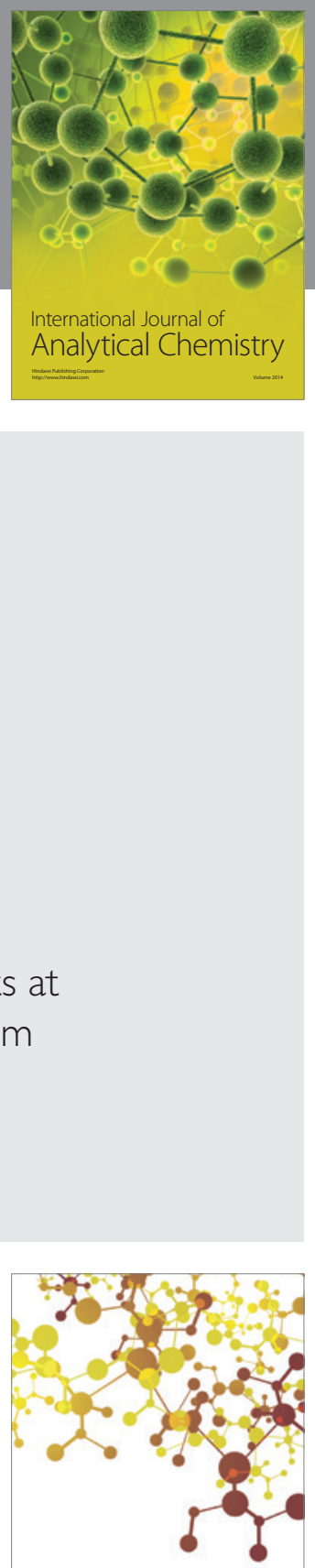

Journal of

Applied Chemistry
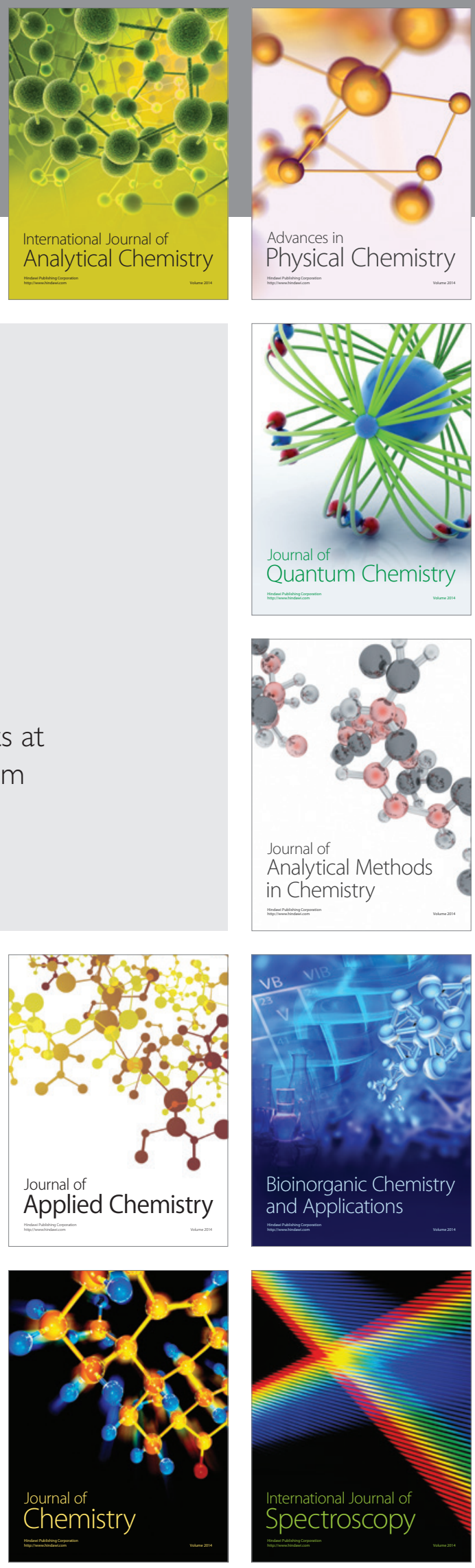